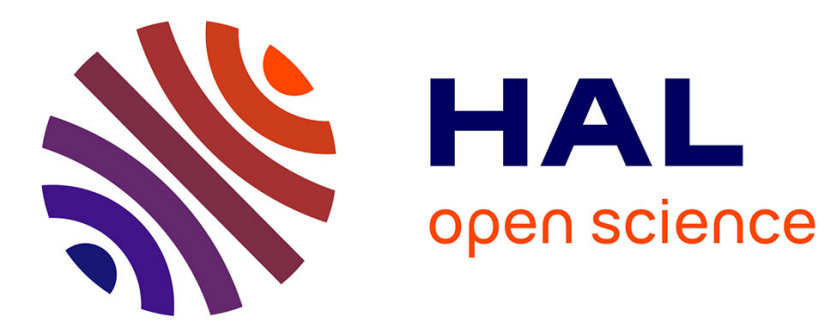

\title{
Les exigences en eau du Frêne (Fraxinus excelsior L.)
}

Gilbert Aussenac, Gérard Lévy

\section{To cite this version:}

Gilbert Aussenac, Gérard Lévy. Les exigences en eau du Frêne (Fraxinus excelsior L.). Revue forestière française, 1992, 44 (S), pp.32-38. 10.4267/2042/26379 . hal-03444109

\section{HAL Id: hal-03444109 \\ https://hal.science/hal-03444109}

Submitted on 23 Nov 2021

HAL is a multi-disciplinary open access archive for the deposit and dissemination of scientific research documents, whether they are published or not. The documents may come from teaching and research institutions in France or abroad, or from public or private research centers.
L'archive ouverte pluridisciplinaire HAL, est destinée au dépôt et à la diffusion de documents scientifiques de niveau recherche, publiés ou non, émanant des établissements d'enseignement et de recherche français ou étrangers, des laboratoires publics ou privés. 


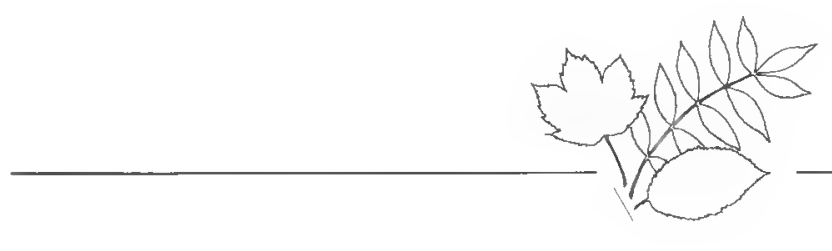

\title{
LES EXIGENCES EN EAU DU FRÊNE (Fraxinus excelsior L.)
}

\author{
G. AUSSENAC - G. LÉVY
}

Parmi les solutions proposées pour la mise en valeur des taillis et des taillis-sous-futaie de la moitié Nord de la France, il est envisagé de favoriser parallèlement au Chêne d'autres espèces, notamment les feuillus précieux, et en particulier le Frène (Fraxinus excelsior L.). Une étude des relations stationproduction (Devauchelle et Lévy, 1977) a mis en évidence le rôle fondamental et même unique des conditions d'approvisionnement en eau pour la croissance du Frêne dans le Nord-Est de la France. Des conclusions tout à fait comparables s'appliquent à la région Nord-Picardie (Le Goff et Lévy, 1984). Afin de pouvoir expliquer les résultats de ces études et d'en généraliser les enseignements, il est nécessaire de disposer d'informations sur les exigences hydriques du Frêne. En fait, peu de travaux ont été réalisés dans ce domaine, et nous nous appuierons pour l'essentiel sur les travaux de Aussenac et Lévy (1983) pour essayer de faire le point sur le comportement de cette espèce en situation de sécheresse. Afin de mieux dégager les particularités du Frêne, nous le comparerons au Chêne pédonculé (Quercus pedunculata Ehrl.) qui lui est proche au plan écologique.

\section{ÉVOLUTION DE L'ÉTAT HYDRIQUE DU FRÊNE EN SITUATION DE SÉCHERESSE. COMPARAISON AVEC LE CHÊNE PÉDONCULÉ}

L'étude a été menée en cases de végétation (Aussenac et Lévy, 1983) et a consisté à étudier l'ècophysiologie de plants de Frêne (âgés de 2 ans) et de Chêne pédonculé (âgés de 3 ans), soumis ou non à une sécheresse; au moment de l'expérimentation, le couvert était totalement fermé. Le protocole expérimental comportait :

- des plants maintenus en permanence à la capacité au champ par un arrosage régulier ;

- des plants soumis à un dessèchement et réhydratés lorsqu'ils ont atteint un potentiel hydrique de base de - 2,0 MPa. II convient d'ailleurs d'indiquer qu'à ce niveau de stress le Frêne a présenté des signes très nets de flétrissement du feuillage, ce qui n'était pas le cas du Chêne.

Au cours de cette expérimentation, il a été constaté que pendant la phase de sécheresse la diminution du potentiel de base a été beaucoup plus rapide chez le Frêne que chez le Chêne. Le temps mis par 
les deux espèces (dans chacune des cases de végétation) pour atteindre différents niveaux de stress hydrique est indiqué dans le tableau I (ci-dessous). En moyenne, pour atteindre un potentiel de base de - 1,0 MPa, les frênes ont mis 29 jours, contre 69 jours pour les chênes. II y a lieu d'ajouter que, compte tenu de cette vitesse d'évolution du potentiel de base, les frênes ont dû être réhydratés trois fois, alors que les chênes ne l'ont été qu'une fois, en fin d'été. Bien qu'il ne soit pas possible de faire une moyenne des consommations en eau, puisque les réalimentations n'ont pas èté effectuées exactement au même niveau de stress hydrique, il est intéressant de noter que toutes les cuves de frênes ont consommé beaucoup plus d'eau que les cuves de chêne (tableau II, ci-dessous).

Tableau I Temps (jour) mis pour atteindre un niveau de stress déterminé chez le Frêne et le chêne

(à partir du début du dessèchement le 22-4-1981)

\begin{tabular}{|c|c|c|c|c|c|}
\hline \multicolumn{3}{|c|}{ Frêne } & \multicolumn{3}{c|}{ Chêne } \\
\hline \multirow{2}{*}{$N^{\circ}$ cuve } & \multicolumn{2}{|c|}{ PB (MPa) } & \multirow{2}{*}{ No Cuve } & \multicolumn{2}{c|}{ PB (MPa) } \\
\cline { 2 - 3 } & $-1,0$ & $-2,0$ & & 68 & 84 \\
\cline { 3 - 6 } 2 & 18 & 25 & 4 & 58 & 71 \\
3 & 30 & 37 & 5 & 64 & 80 \\
11 & 37 & 39 & 8 & 53 & 75 \\
12 & 31 & 37 & 9 & 75 & \\
13 & 31 & 37 & 10 & 81 & \\
14 & 18 & 26 & 17 & 81 & \\
15 & 23 & 31 & 18 & 88 & \\
16 & 31 & 37 & 19 & 53 & 84 \\
20 & 31 & 37 & 21 & 71 & 81 \\
25 & 37 & 40 & 23 & $\mathbf{6 9 , 2}$ & \\
\hline
\end{tabular}

Tableau II

Consommation en eau des cuves de Frêne et de Chêne pendant la période du 22-4-1981 au 28-9-1981

\begin{tabular}{|c|c|c|c|c|c|}
\hline \multicolumn{3}{|c|}{ Frêne } & \multicolumn{3}{c|}{ Chêne } \\
\hline$N^{\circ}$ de cuve & Traitement & $\begin{array}{c}\text { Consommation } \\
\text { en eau (I) }\end{array}$ & $N^{\circ}$ de cuve & Traitement & $\begin{array}{c}\text { Consommation } \\
\text { en eau (I) }\end{array}$ \\
\hline 1 & $\begin{array}{c}\text { Capacité } \\
\text { au champ } \\
\text { Capacité }\end{array}$ & 1517 & 7 & $\begin{array}{c}\text { Capacité } \\
\text { au champ } \\
\text { Capacité }\end{array}$ & 1350 \\
24 & $\begin{array}{c}\text { au champ } \\
\text { au champ }\end{array}$ & 1200 \\
2 & Dessèchement & 710 & 22 & Dessèchement & 430 \\
3 & Dessèchement & 813 & 4 & Dessèchement & 495 \\
11 & Dessèchement & 736 & 8 & Dessèchement & 489 \\
12 & Dessèchement & 745 & 9 & Dessèchement & 512 \\
14 & Dessèchement & 802 & 10 & Dessèchement & 482 \\
15 & Dessèchement & 730 & 17 & Dessèchement & 437 \\
16 & Dessèchement & 791 & 18 & Dessèchement & 527 \\
20 & Dessèchement & 824 & 19 & Dessèchement & 461 \\
$25 *$ & Dessèchement & 942 & 21 & Dessèchement & 516 \\
Dessèchement & 624 & 23 & Dessèchement & 537 \\
\hline
\end{tabular}

(") Arbres atteints par les gelées tardives et trés retardés dans leur développement. 
En situation d'alimentation optimale en eau (capacité au champ), le Frêne a eu une consommation supérieure au Chêne mais ces différences ont été beaucoup moins importantes que dans le cas des cuves en dessèchement.

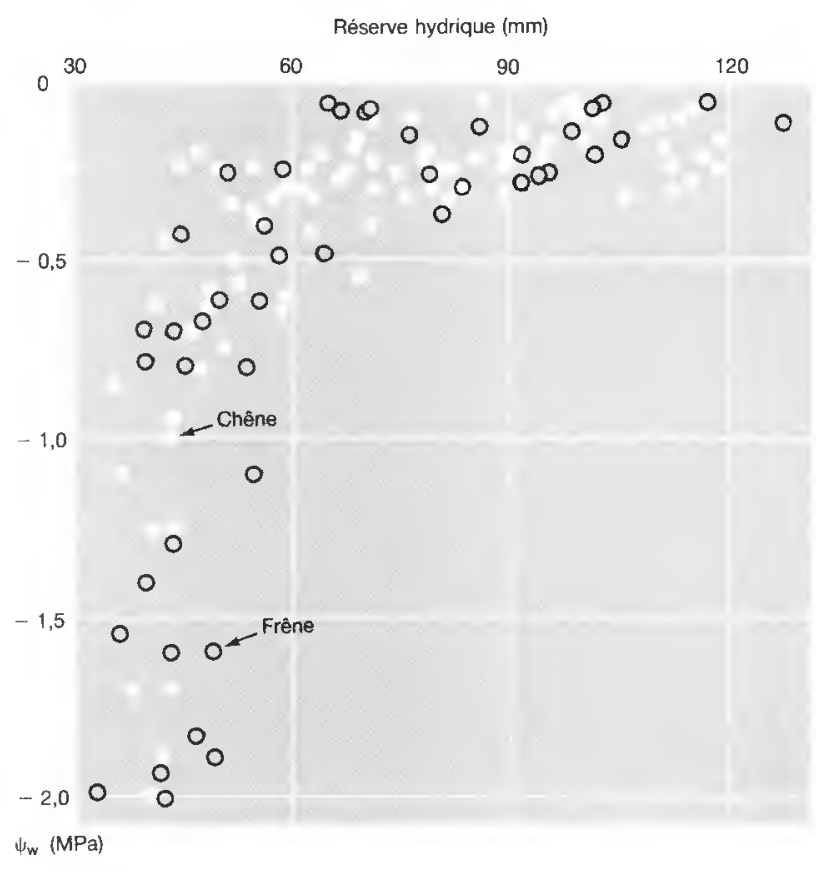

De fait, en raison de la texture sableuse du sol, le potentiel de base des plants n'a commencé à baisser véritablement que lorsque la réserve hydrique du sol est devenue inférieure à $45 \%$ de la réserve à la capacité au champ (figure 1 , ci-contre). La relation est identique pour les deux espèces mais, en réalité, la vitesse d'exploitation de la réserve hydrique a été plus rapide pour le Frêne.

Figure 1

RELATION ENTRE LA RÉSERVE EN EAU DU SOL $(\mathrm{mm})$ ET LE POTENTIEL DE BASE (MPa) DES ARBRES

\section{INFLUENCE DE LA DISPONIBILITÉ EN EAU SUR LA CONDUCTANCE STOMATIQUE ET L'ÉVAPOTRANSPIRATION, COMPARAISON AVEC D'AUTRES ESPĖCES FEUILLUES}

Les résultats précédents montrent que le Frêne consomme beaucoup plus rapidement l'eau disponible dans le sol que le Chêne. Afin d'essayer de comprendre ce résultat dans une première approche et en phase de dessèchement, on a étudié la relation entre l'évapotranspiration réelle et la réserve hydrique du sol (figure 2, p. 35). On constate que le Frêne ne diminue sensiblement sa transpiration que lorsque les réserves hydriques sont devenues faibles; comparativement le Chêne la diminue fortement dès que le sol se dessèche légèrement. Ce phénomène explique que le Frêne consomme beaucoup plus rapidement les réserves en eau que le Chêne. D'une façon générale, pour l'ensemble de la période d'étude, on constate que le Frêne ne commence à diminuer son évapotranspiration que lorsque la réserve en eau du sol est très abaissée (environ $60 \%$ de la réserve à la capacité au champ), alors que pour le Chêne une forte baisse est déjà acquise pour une réserve encore élevée (inférieure de $13 \%$ à la capacité au champ).

Une approche plus fine par l'étude des relations existant au cours d'une journée (9 juillet 1981) entre potentiel hydrique $(\Psi)$ et résistance stomatique ( $r s$ ) explique bien les résultats précédents et fait apparaître un comportement très différent des deux espèces (figure 3, p. 35). En effet, pour une même chute du potentiel hydrique, la résistance stomatique augmente plus vite chez le Chêne que chez le Frêne. On constate aussi que lorsque le potentiel a atteint $-1,4 \mathrm{MPa}$ chez le Chêne la résistance stomatique a augmenté considérablement, alors que cela ne s'est produit chez le Frêne que pour des valeurs comprises entre $-3,0$ et $-3,5 \mathrm{MPa}$. 


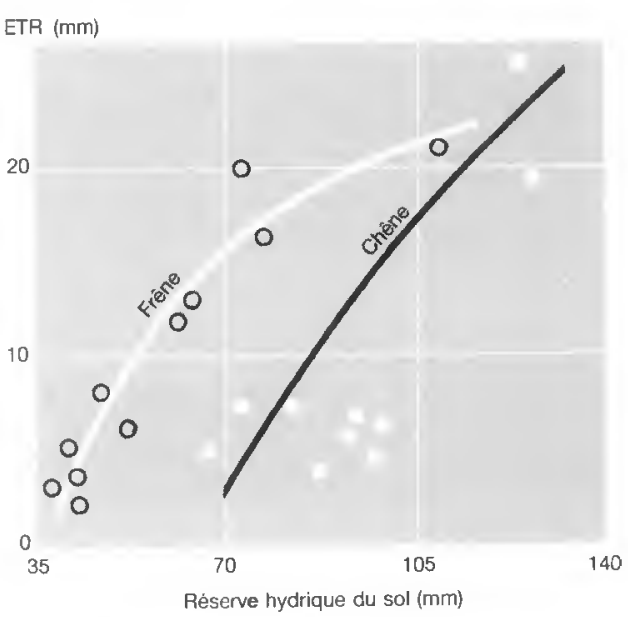

Figure 2

RELATION ENTRE L'ÉVAPOTRANSPIRATION RÉELLE (ETR) ET LA RÉSERVE EN EAU DU SOL DANS LES DIFFÉRENTES CUVES ÉTUDIÉES

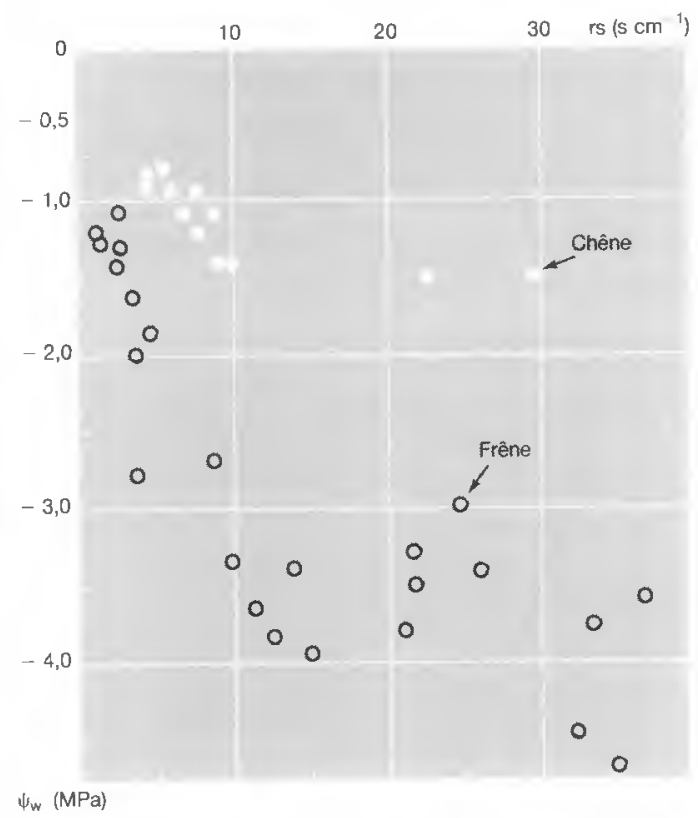

Figure 3

RELATION ENTRE LA RÉSISTANCE STOMATIQUE (rs) ET LE POTENTIEL HYDRIQUE ( $\psi$ ) DES RAMEAUX DE CHÊNE

ET DE FRÊNE

\section{INFLUENCE DU DESSĖCHEMENT DU SOL SUR LA CROISSANCE EN HAUTEUR}

L'évolution de l'accroissement cumulé en hauteur est rapporté sur la figure 4, ci-dessous. Le Frêne n'a eu qu'une seule phase de croissance, alors que le Chêne en a eu trois dans le traitement à la capacité au champ et deux pour les cuves subissant un dessèchement. Dans le cas d'une alimentation en eau optimale (capacité au champ), les frênes ont eu une croissance en hauteur plus forte que les chênes, mais ces derniers ont des ramifications latérales beaucoup plus nombreuses et denses qui font que, au total, la biomasse foliaire

Figure 4 ÉVOLUTION COMPARÉE DE L'ALLONGEMENT MOYEN CUMULÉ DE LA POUSSE TERMINALE DES PLANTS Á LA CAPACITÉ AU CHAMP (cc) ET OES PLANTS SOUMIS Á LA SÉCHERESSE (s)

80 Allongement moyen cumulé (cm)

60

20

40

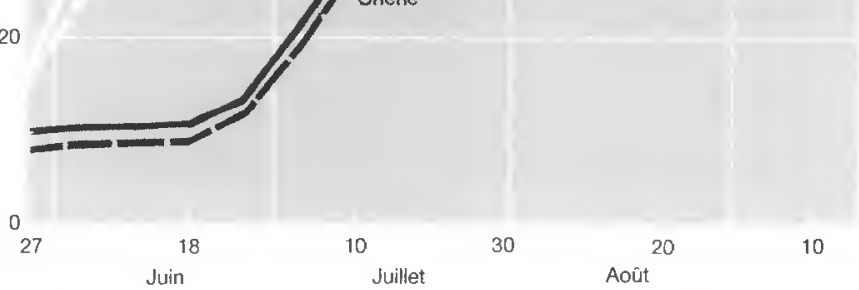


des deux espèces apparait très comparable. Les arbres soumis au dessèchement ont moins poussé que les arbres régulièrement bien arrosés.

L'examen de l'allongement relatif des pousses en fonction du potentiel de base (figure 5, ci-dessous) montre que la croissance en hauteur du Frêne s'est arrêtée lorsque le potentiel hydrique de base s'est abaissé en dessous de - 1,1 MPa. Le Chêne pédonculé a un comportement identique; mais il y a lieu de remarquer qu'en fait le Frêne, compte tenu de son taux élevé de transpiration, atteint plus précocement ce niveau de stress.

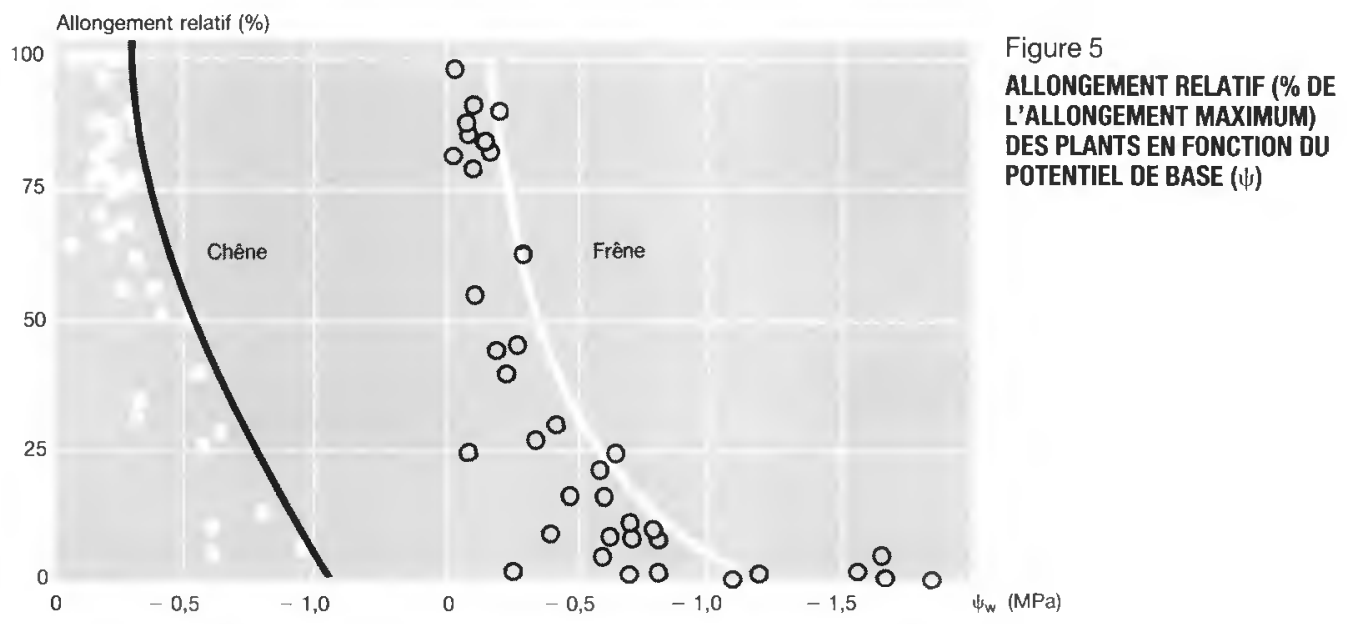

Tableau III Comparaison du Frêne avec d'autres feuillus de régions tempérées (d'après Besnard et Carlier, 1990)

\begin{tabular}{|c|c|c|c|c|}
\hline \multirow{2}{*}{ Espèces } & \multirow{2}{*}{ Localisation } & \multicolumn{2}{|c|}{ Transpiration maximale } & \multirow{2}{*}{$\begin{array}{l}\text { Conductance } \\
\text { hydraulique } \\
\mathrm{mol} / \mathrm{m}^{2} / \mathrm{s} / \mathrm{Pa}\end{array}$} \\
\hline & & $\mathrm{g} / \mathrm{m}^{2} / \mathrm{s}$ & $\mathrm{g} / \mathrm{m}^{2} / \mathrm{j}$ & \\
\hline $\begin{array}{l}\text { Acer negundo } \\
\text { Acer saccharum } \\
\text { Alnus incana } \\
\text { Betula pendula } \\
\text { Betula pendula }\end{array}$ & $\begin{array}{l}\text { USA } \\
\text { USA } \\
\text { France, forêt alluviale } \\
\text { Danemark } \\
\text { Autriche }\end{array}$ & $\begin{array}{c}0,12-0,22 \\
0,064 \\
0,044\end{array}$ & $\begin{array}{r}640 \\
1220\end{array}$ & \\
\hline $\begin{array}{l}\text { Betula verrucosa } \\
\text { Castanea sativa } \\
\text { Fagus sylvatica } \\
\text { Fagus sylvatica }\end{array}$ & $\begin{array}{l}\text { Salle conditionnée } \\
\text { France, Dauphiné } \\
\text { Danemark } \\
\text { Autriche }\end{array}$ & $\begin{array}{c}0,09-0,015 \\
0,038 \\
0,024\end{array}$ & $\begin{array}{c}2250 \\
2740-4000 \\
600-1400 \\
976\end{array}$ & $5.3 \times 10^{-9}$ \\
\hline $\begin{array}{l}\text { Fraxinus excelsior } \\
\text { Fraxinus excelsior } \\
\text { Liriodendron tulipifera } \\
\text { Populus tremula }\end{array}$ & $\begin{array}{l}\text { Danemark } \\
\text { France, forêt alluviale } \\
\text { Salle conditionnée }\end{array}$ & $\begin{array}{c}0,072 \\
0,10-0,22\end{array}$ & $\begin{array}{c}1200-2500 \\
3000-5000 \\
1178 \\
2000\end{array}$ & $10,8 \times 10^{-9}$ \\
\hline $\begin{array}{l}\text { Populus sp. } \\
\text { Quercus alba }\end{array}$ & $\begin{array}{l}\text { Wisconsin } \\
\text { Durham, NC }\end{array}$ & & 1421 & $\begin{array}{r}4,0-5,3 \times 10^{-9} \\
1,54 \times 10^{-9}\end{array}$ \\
\hline $\begin{array}{l}\text { Quercus rubra } \\
\text { Quercus petraea } \\
\text { Salix cinerea }\end{array}$ & $\begin{array}{l}\text { Durham, NC } \\
\text { Danemark } \\
\text { France, forêt alluviale }\end{array}$ & $\begin{array}{c}0,061 \\
0,07-0,16\end{array}$ & $\begin{array}{r}1202-1245 \\
900-2300\end{array}$ & \\
\hline
\end{tabular}




\section{INTERPRÉTATION GLOBALE DU COMPORTEMENT DU FRÊNE VIS-À-VIS DE LA DISPONIBILITÉ EN EAU; CONSÉQUENCES POUR LA SYLVICULTURE}

Le Frêne peut donc être considéré comme une espèce grosse consommatrice d'eau dans la mesure où elle ne contrôle que très tardivement sa transpiration. Ce comportement a pour conséquence, toutes choses égales par ailleurs, d'amener cette espèce à des stress hydriques très élevés et ceci beaucoup plus rapidement par exemple que le Chêne pédonculé qui est une espèce écologiquement voisine. Dans I'hypothèse d'une alimentation en eau déficiente, le Frêne se trouve donc beaucoup plus précocement en position difficile que le Chêne.

Cette capacité transpiratoire élevée du Frêne a été également notée par Braun (1977), qui trouve que cette espèce a une transpiration plus élevée que l'Aulne glutineux et les grands Érables. Besnard et Carlier (1990) concluent (tableau III, ci-dessous) que le Frêne présente des transpirations maximales bien supérieures à celles d'autres arbres tempérés. Cette caractéristique est pour une part liée à une conductance stomatique élevée mais aussi à une conductance hydraulique forte, c'est-à-dire à une faible résistance. au transfert de l'eau en phase liquide dans l'arbre depuis les racines jusqu'aux feuilles.

A ce sujet ces mêmes auteurs constatent que, en situation de plaine alluviale (nappe phréatique toujours présente à faible profondeur) malgré une très forte transpiration, le potentiel hydrique minimum (atteint en milieu de journée) du Frêne ne tombe pas en dessous de $-1,9 \mathrm{MPa}$ (figure 6 , ci-contre).

Kramer et Kozlowski, 1960

Kramer et Kozlowski, 1960

Besnard, 1987

Ladefoged, 1963

Kramer et Kozlowski, 1960

Jarvis et Jarvis, 1963

Collicard, 1982

-adefoged, 1963

<ramer et Kozlowski, 1960

3esnard et Carlier, 1990

<ramer et Kozlowski, 1960

Jarvis et Jarvis, 1963

Jallardy et Kozlowski, 1981

<ramer et Kozlowski, 1960

finckley et Bruckerhoff, 1975

íramer et Kozlowski, 1960

_adefoged, 1963

3esnard, 1987
Figure 6

COMPARAISON DE L'ÉVOLUTION JOURNALIËRE (HEURES TU)

DES PARAMĖTRES

MICROMÉTÉORO-

LOGIQUES (EN BAS)

ET PHYSIOLOGIQUES

(EN HAUT) AU COURS

D'UNE BELLE JOURNÉE

D'ÉTÉ CHEZ LE FRÊNE

Q : rayonnement photosynthétiquement actif $\left(\mu\right.$ moles $\left.\mathrm{m}^{2} \mathrm{~s}^{1}\right)$

$\mathrm{HR}$ : humidité relative de l'air (\%)

DPV: déficit de pression de vapeur ( $\mathrm{kPa}$ )

t : temperature de l'air $\left({ }^{\circ} \mathrm{C}\right)$

O $\mathrm{Tr}$ : transpiration (gs ${ }^{1} \mathrm{~m}^{2}$ )

Ogs: conductance stomatique $\left(\mathrm{m}^{\mathrm{s}}\right)$ pour la vapeur d'eau, potentiel hydrique (MPa)
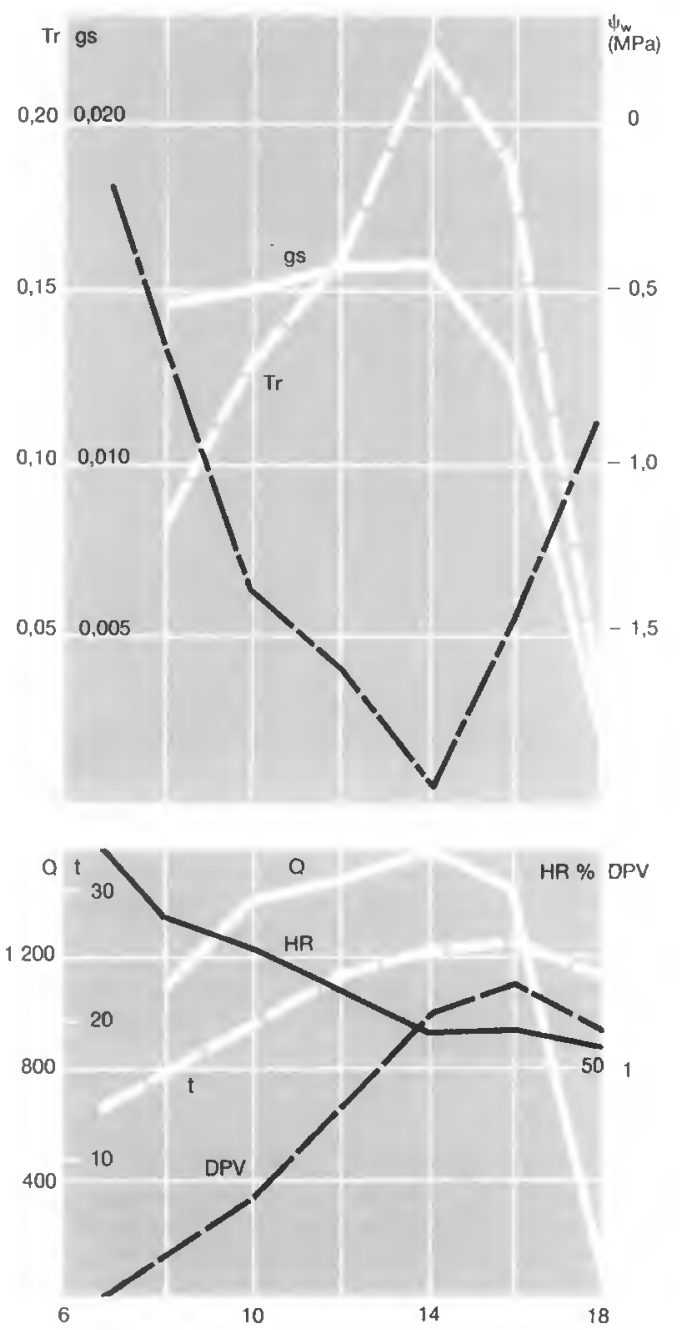


\section{G. AUSSENAC - G. LÉVY}

Kozlowski et al. (1974), étudiant Acer saccharum et Fraxinus americana, observent aussi une transpiration élevée du Frêne américain ; ils attribuent cette capacité transpiratoire importante à des stomates plus grands, mais peu nombreux. Les comptages effectués dans le cas de Fraxinus exce/sior (175 stomates $/ \mathrm{mm}$ ) correspondent à des chiffres très inférieurs au Chêne pédonculé ( 500 stomates $/ \mathrm{mm}$ ), mais il ne semble pas y avoir de grandes différences en ce qui concerne la taille des stomates. L'arrêt de la croissance en hauteur chez le Frêne ne se produit pas à un niveau de stress hydrique plus faible $(-1,1 \mathrm{MPa})$ que le Chêne pédonculé mais il intervient plus précocement dans le temps.

Au plan sylvicole, les résultats obtenus ici permettent de préciser les limites du Frêne. II s'agit d'une espèce dont l'utilisation ne peut être envisagée que dans des situations à disponibilités en eau importantes: présence d'une nappe phréatique, sol profond ou bas de pente. Dans les zones à bilan hydrique déficitaire fréquent, l'avenir des arbres est compromis. Ces résultats recoupent les travaux de terrain qui indiquent qu'en Lorraine l'alimentation en eau est le principal facteur limitant la production du Frêne. Notons aussi qu'en peuplement, il est classique d'observer sur les sols peu profonds de plateaux calcaires des régénérations importantes de Frêne qui disparaissent rapidement. Dans la mise en valeur des taillis et taillis-sous-futaie, il est certain que le Frêne ne peut être envisagé que dans des situations bien particulières.

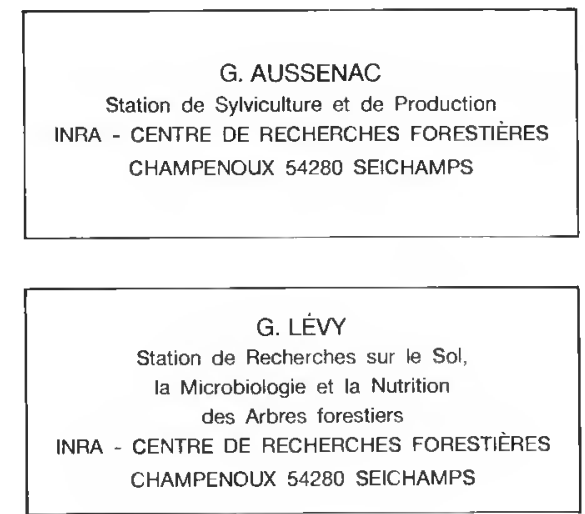

\section{BIBLIOGRAPHIE}

AUSSENAC (G.), GRANIER (A.). - Quelques résultats de cinétique journalière du potentiel de sève chez les arbres forestiers. - Annales des Sciences forestières, vol. 35, $\mathrm{n}^{\circ} 1,1978, \mathrm{pp} .19-32$.

AUSSENAC (G.), LÉVY (G.). - Influence du dessèchement du sol sur le comportement hydrique et la croissance du Chêne pédonculé (Quercus pedunculata Ehrl.) et du Frêne (Fraxinus excelsior L.) cultivés en case de végétation. Annales des Sciences forestieres, vol. 40, $n^{\circ} 3,1983$, pp. 251-264.

BESNARD (G.), CARLIER (G.). - Potentiel hydrique et conductance stomatique des feuilles de frêne (Fraxinus excelsior L.) dans une forêt alluviale du Haut-Rhône français. - Annales des Sciences forestières, vol. 47, $n^{\circ} 4$, 1990, pp. 353-365.

BRAUN (H.J.). - Growth and water economy of the trees Acer platanoides L., Acer pseudoplatanus L., and Fraxinus excelsior L. - Z. Pflanzenphysiol., vol $84, \mathrm{n}^{\circ} 5,1977$, pp. 459-462.

DEVAUCHELLE (R.), LÉVY (G.). - Propriétés stationnelles et croissance du Frêne dans l'Est de la France, étude de certaines caractéristiques de cette essence. - Annales des Sciences forestières, vol. 34, $\mathrm{n}^{\circ} 3,1977, \mathrm{pp} .231-244$.

KOZLOWSKI (T.T.), DAVIES (W.J.), CARLSON (S.D.). - Transpiration rates of Fraxinus americana and Acer saccharum leaves. - Canadian Journal of Forest Research, vol. 3, n' 4, 1974, pp. 259-267.

LE GOFF (N.), LÉW (G.). - Productivité du Frêne (Fraxinus excelsior L.) en région Nord-Picardie. B - Étude des relations entre la productivite et les conditions de milieu. - Annales des Sciences forestières, vol. $41, n^{\circ} 2,1984$, pp. 135-170. 